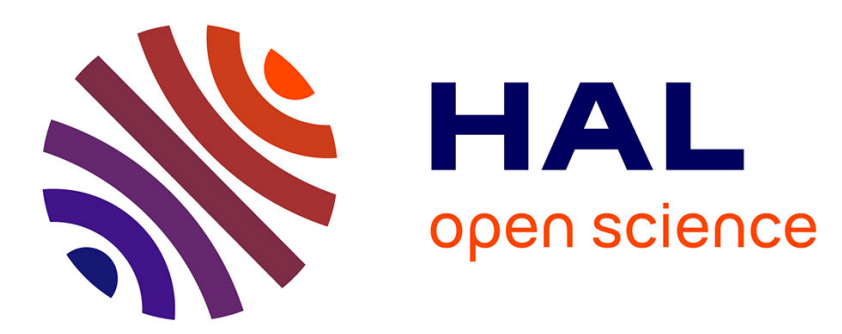

\title{
Identification de deux espèces de rongeurs de Guyane française, Proechimys cuvieri et Proechimys guyannensis (Echimyidae) par l'analyse des correspondances
}

\author{
Michel Guillotin, Jean-François Ponge
}

\section{- To cite this version: \\ Michel Guillotin, Jean-François Ponge. Identification de deux espèces de rongeurs de Guyane française, Proechimys cuvieri et Proechimys guyannensis (Echimyidae) par l'analyse des correspondances. Mam- malia, 1984, 48 (2), pp.287-291. 10.1515/mamm.1984.48.2.287 . hal-00507085}

\section{HAL Id: hal-00507085 \\ https://hal.science/hal-00507085}

Submitted on 13 Jan 2011

HAL is a multi-disciplinary open access archive for the deposit and dissemination of scientific research documents, whether they are published or not. The documents may come from teaching and research institutions in France or abroad, or from public or private research centers.
L'archive ouverte pluridisciplinaire HAL, est destinée au dépôt et à la diffusion de documents scientifiques de niveau recherche, publiés ou non, émanant des établissements d'enseignement et de recherche français ou étrangers, des laboratoires publics ou privés. 
Identification de deux espèces de rongeurs de Guyane. frantçaise, Proechimys cuvieri et Proechimys guyannensis (Echimyidae) par l'analyse

\title{
des correspondances
}

\author{
par M. GUILLOTIN et J.F. PONGE \\ Laboratoire d'Ecologie Générale,
}

Muséum National d'Histoire Naturelle, 91800 Brunoy

Deux espèces de rongeurs de Guyane française, Proechimys cuvieri et Proechimys guyannensis, ne peuvent être différenciées avec certitude grâce aux méthodes morphologiques classiques. Seule une analyse des correspondances a permis de séparer les deux espèces grâce à certaines mensurations crâniennes: longueur des molaires et largeur du trou occipital.

Petter (1978) a montré que l'on trouve en Guyane française deux espèces sympatriques de Proechimys différant par leurs mensurations et leur morphologie. Proechimys cuvieri est l'espèce la plus grande; la longueur de la rangée dentaire supérieure est comprise entre 8,1 et $9,3 \mathrm{~mm}$ contre 7 à $8,5 \mathrm{~mm}$ chez Proechimys guyannensis et chez la première espèce la deuxième dent de la mandibule a le plus souvent 3 îlots d'émail au lieu de 2 chez la seconde. L'existence de ces deux espèces a été confirmée par une étude chromosomique qui attribue à $P$. cuvieri $2 \mathrm{~N}=28$ chromosomes et à $P$. guyannensis $2 \mathrm{~N}=40$ chromosomes.

On constate donc qu'il existe un certain recouvrement dans les mensurations de ces deux espèces; de surcroît le nombre dîlots d'émail est parfois difficile à déterminer, particulièrement chez les jeunes animaux dont un des îlots est souvent bifide et, à l'inverse, chez les plus vieux où le dessin dentaire est effacé. Dans la pratique, on ne peut donc ranger à coup sûr chaque individu dans l'une ou l'autre espèce au seul vu des critères morphologiques précités. Nous avons donc recherché un critère systématique absolu.

Les 90 spécimens étudiés dans ce travail ont été récoltés en Guyane française et sur chaque individu ont été effectuées 34 mensurations corporelles et crâniennes. 
La méthode d'analyse multivariée utilisée est l'analyse des correspondances de Benzecri (Lebart et al., 1979). Rappelons brièvement qu'il s'agit d'une méthode permettant une représentation géométrique de l'ensemble des individus placés selon leurs affinités biométriques. Elle procède de la décomposition de la variance totale en facteurs linéaires indépendants. Chaque facteur est figuré par un axe d'inertie du nuage de points représentant l'ensemble des individus, passant par le centre de gravité. Les premiers axes d'inertie (facteurs) correspondent à l'essentiel de l'information contenue dans les données. Ici, le premier facteur extrait $68,5 \%$ de la variance totale, le second $10,3 \%$ soit un total de $78,8 \%$ à eux deux. La projection simultanée des variables et des individus sur ces axes factoriels enrichit l'interprétation. Les individus sont situés dans la même direction que les variables qui les caractérisent le plus. Les 34 variables ont été centrées et réduites $(\mathrm{x}=10, \sigma=1)$, afin d'éviter des dispersions gênantes dans le plan dues à l'hétérogénéité très grande des variances et des moyennes. Ceci a permis en outre d'avoir une représentation directe des contributions relatives, puisque le carré de la distance d'un point-variable par rapport à l'origine se trouve le même pour tous les paramètres. Un point-variable le long d'un axe sera d'autant plus éloigné de l'origine que sa contribution relative à cet axe sera grande, donc qu'il sera «explicatif». Les variables ont été dédoublées par complémentarité à 20, afin de représenter par un point la valeur faible de chaque mesure et de faire ressortir le facteur taille. Les données manquantes (crânes incomplets et jeunes sans deuxième et troisième molaires) ont été simulées selon la méthode préconisée par Benzecri et al. (1973) à partir d'une analyse portant sur les données non centrées, non réduites et non dédoublées (données brutes).

C'est une représentation dans le plan des 2 premiers axes qui a été choisie (fig. 1).

L'axe 1 représente un gradient de taille. On trouve, le long de cet axe, toutes les mensurations liées à l'âge des animaux: mensurations corporelles (longueurs de la tête et du corps: $\mathrm{T}+\mathrm{C}$, de la patte postérieure: ppp, et de la queue: qqq) et la plupart des mensurations crâniennes (longueur condylo-basale: lcb, longueur du diastème: dil, longueur naso-sagittale: fll, etc.). Les points situés à droite de l'axe des abscisses représentent les vieux individus, alors qu'à gauche on trouve les jeunes spécimens.

L'axe 2 oppose les animaux selon les dimensions des 3 premières dents jugales (longueur et largeur de la prémolaire et des deux premières molaires: $\mathrm{mL} 1, \mathrm{ml} 1, \mathrm{~mL} 2, \mathrm{ml} 2, \mathrm{~mL} 3, \mathrm{ml} 3$ ) et selon la largeur du trou occipital (toi); les valeurs les plus grandes sont en haut et les plus petites en bas. En dehors de la taille, ces critères sont les plus discriminants pour séparer les animaux, ce qui correspond plus ou moins à la longueur totale utilisée empiriquement par Petter pour différencier les deux espèces; mais l'adjonction de la deuxième et surtout la troisième molaire rend, nous l'avons vu, la séparation spécifique moins évidente. 
En représentant (fig. 2) 5 de ces paramètres les plus représentatifs (longueur et largeur de la PM1 + longueur et largeur de la M1 en fonction de la largeur du trou occipital) et en indiquant sur la figure les individus dont on connaît le nombre de chromosomes ( 1 cercle pour $P$. cuvieri, 2 cercles pour $P$. guyannensis), ainsi que ceux qui possèdent 2 ou 3 îlots d'émail sur la première molaire inférieure (soulignés respectivement de 1 ou 2 traits), on remarque que tous les $P$. cuvieri déterminés avec certitude se retrouvent en haut à droite et les $P$. guyannensis en bas à gauche. Une droite tracée arbitrairement entre ces deux lots permet de séparer les individus des deux espèces sans qu'il y ait de recouvrement.

Pour identifier un Proechimys de Guyane, 5 mensurations crâniennes sont donc nécessaires (longueurs et largeurs de PM1 et M1 et largeur du trou occipital) et cela quel que soit son âge.

Parmi les 90 spécimens étudiés ici, 16 soit 17,7\% sont référables à $P$. guyannensis et $74(82,3 \%)$ à $P$. cuvieri, ce qui confirme la faible représentativité de la première espèce dans les milieux qui ont été prospectés en Guyane française.

Dans un précédent travail (Guillotin, 1982), nous avions indiqué que sur 73 Proechimys de Guyane étudiés avec les critères utilisables jusqu'à ce jour (principalement le dessin dentaire), seulement 8,2\% des individus pouvaient être attribués à $P$. guyannensis. La différence entre ce chiffre et celui que nous proposons ici provient du fait que nous n'avions pu identifier la majorité des jeunes individus chez lesquels un des îlots d'émail de la première molaire est souvent bifide et que nous nous étions abstenu de les attribuer à l'une ou l'autre espèce.

Parmi les $P$. guyannensis capturés en Guyane, nous avons pu retrouver le biotope exact de capture de 12 d'entre eux, Il apparaît que les animaux proviennent de toutes les zones prospectées: forêts primaire et secondaire, bordures et zones plus ou moins inondables. Il en est de même pour $P$. cuvieri. Pourtant la majorité des $P$. guyannensis (8 sur 12) proviennent de forêts secondaires plus ou moins âgées, alors que les captures de $P$. cuvieri ont été effectuées indifféremment dans tous les milieux. D'après ces renseignements, bien qu'il soit difficile d'attribuer un biotope particulier à chaque espèce, il semble donc que $P$. guyannensis se rencontre surtout dans les zones les plus ouvertes.

Le couple $P$. cuvieri et $P$. guyannensis est à ranger indiscutablement dans la cohorte des espèces dites «jumelles» dont le terme recouvre le plus souvent une insuffisance dans nos moyens actuels de discrimination morphologique. A cet égard, si $P$. cuvieri et $P$. guyannensis peuvent être aisément séparés avec des spécimens 
morts, il n'en est malheureusement pas encore de même lorsque l'on a affaire à des spécimens vivants.

\section{SUMMARY}

Two species of rodents of French Guyana, Proechimys cuvieri and Proechimys guyannensis, are difficult to differentiate certainly with classical methods. Here is tested an analysis of correspondences which allowed to distinguish the two species with some cranial measurements: molars length and occipital hole breadth.

\section{BIBLIOGRAPHIE}

BENZECRI, J.P., et coll., 1973. - L'analyse des données. II. L'analyse des correspondances. Dunod, Paris, 619 p.

Gulllotin, M., 1982. - Place de Proechimys cuvieri (Rodentia, Echimyidae) dans les peuplements micromammaliens terrestres de la forêt guyanaise. Mammalia, 46: 299-318.

Lebart, L., A. Morineau et J.P. Fenelon, 1979. - Traitement des données statistiques. Dunod, Paris, 510 p.

PetTeR, F., 1978. - Epidémiologie de la leishmaniose en Guyane française en relation avec l'existence d'une espèce nouvelle de Rongeurs Echimyidés Proechimys cuvieri sp. n. C. R. Acad. Sci. Paris, Sér. D, 287: $261-264$.

Reig, O.A., M. Tranier et M.A. BARros, 1979. - Sur l'identification chromosomique de Proechimys guyannensis (E. Geoffroy 1803) et de Proechimys cuvieri Petter 1978 (Rodentia, Echimyidae). Mammalia, 43: 501-505. 


\section{Légendes des figures}

Fig. 1. - Plan 1-2 de l'analyse des correspondances. Chaque individu est représenté par un point et chaque variable par son symbole.

Fig. 2. - Longueurs et largeurs des deux premières dents jugales en fonction de la largeur du trou occipital, indiquant la séparation graphique entre les deux espèces. Les points encerclés correspondent aux individus dont on connaît la formule chromosomique et ceux soulignés aux Proechimys sur lesquels on peut observer 2 ou 3 crêtes d'émail (voir texte). 


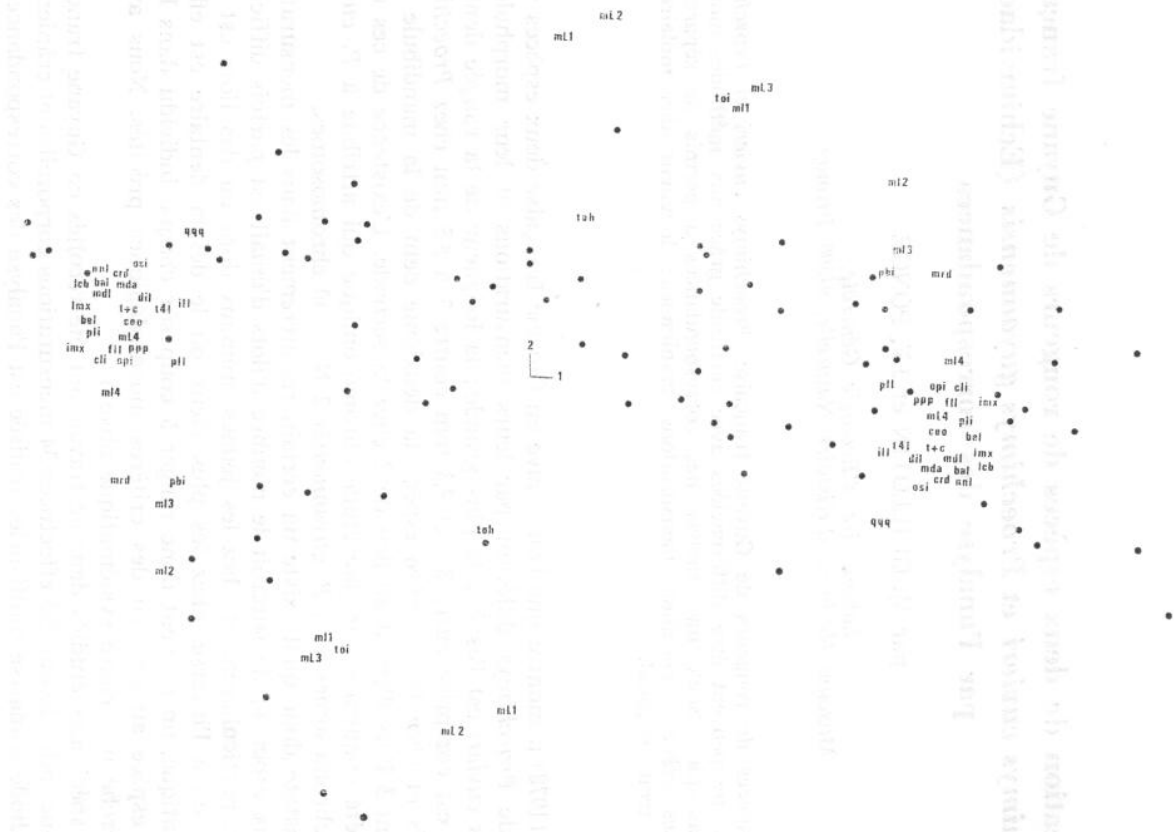

Fig. 1 


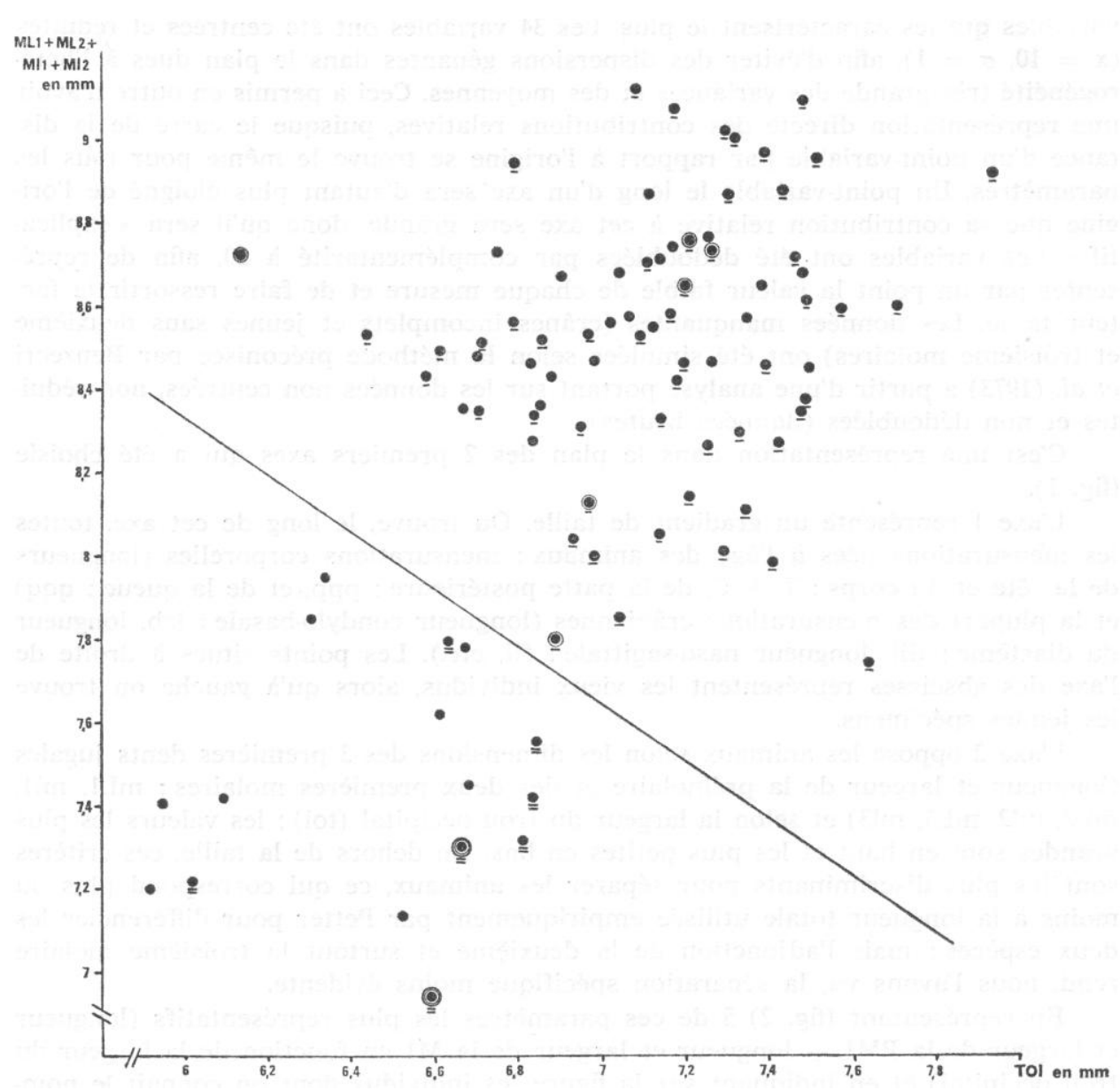

Fig. 2 